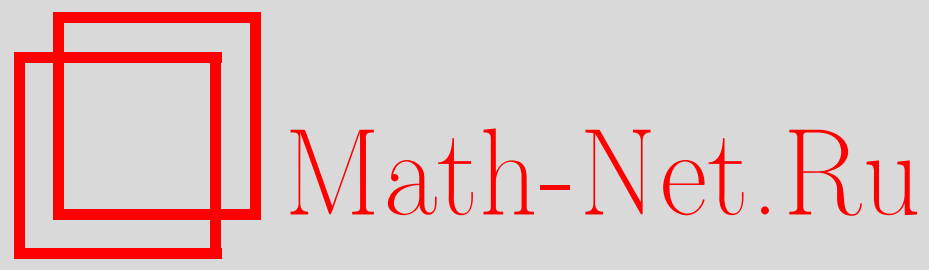

С. А. Вугальтер, Г. М. Жислин, Устойчивость систем большого числа частиц в магнитных полях, Функи. анализ и его прил., 1996, том 30, выпуск 2, 70-73

DOI: https://doi.org/10.4213/faa522

Использование Общероссийского математического портала Math$\mathrm{Net.Ru}$ подразумевает, что вы прочитали и согласны с пользовательским соглашением http://www . mathnet.ru/rus/agreement

Параметры загрузки:

IP : 52.23 .180 .231

26 апреля 2023 г., 11:45:52

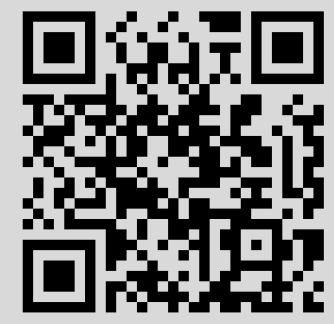




\title{
Устойчивость систем большого числа частиц в магнитных полях
}

\author{
(C) 1996. С. А. ВугАльтеР, Г. М. Жислин ${ }^{1}$
}

В заметке изучается вопрос об устойчивости при $N \rightarrow \infty$ системы $N$ тождественных частиц в магнитном поле. Рассматриваются магнитные поля, имеющие направление оси $z$ и не зависящие от $z$. Кроме того, в случае полей, не возрастающих на бесконечности (в $(x, y)$-плоскости), мы считаем, что поле обладает $S O(2)$-симметрией. Потенциал $V$ парного взаимодействия частиц предполагается знакопеременным, что характерно для внутриядерных взаимодействий (отметим, что в случае знакопостоянных отрицательных потенциалов устойчивость $N$-частичных систем при любом $N$ легко получается из [1]).

Основной результат статьи: устанавливается, что при слабых ограничениях ${ }^{2}$ на потенциал $V$ в магнитном поле существуют стабильные $N$-частичные системы со сколь угодно большим $N$; тем самым результаты об устойчивости многонуклонных систем в отсутствие магнитных полей [2] обобщаются на системы в магнитных полях. При этом мы осуществляем в доказательстве более естественный, чем в [2], подход к учету запрета Паули. Вместо того чтобы рассматривать по отдельности типы симметрии, отвечающие допустимым для данной задачи схемам Юнга, мы рассматриваем все множество таких типов одновременно, т. е. изучаем спектр многочастичного гамильтониана на прямой сумме пространств, отвечающих всевозможным допустимым схемам Юнга. Преимущество данного подхода проявляется в том, что для справедливости утверждения формулируемой теоремы (в отличие от теоремы 2.2 из [2]) не требуется выполнения определенных неравенств между нижними гранями операторов в пространствах различной перестановочной симметрии (таких, например, как неравенство (3.1) из [2])

Рассмотрим квантовую систему $N$ тождественных частиц. Пусть $r_{i}=$ $\left(x_{i}, y_{i}, z_{i}\right), m, e$ - соответственно радиус-вектор, масса и заряд $i$-й частицы, $\rho_{i}=\left(x_{i}, y_{i}\right), A_{i}=\left(A_{\perp}\left(\rho_{i}\right), 0\right)=\left(A_{1}\left(\rho_{i}\right), A_{2}\left(\rho_{i}\right), 0\right)$ - магнитный векторпотенциал и $V\left(r_{i j}\right)$ — потенщиал взаимодействия частиц с номерами $i$ и $j$ между собой. Относительно потенциала $V(\xi), \xi \in \mathbb{R}^{3}$, будем предполагать, что $V(\xi)=V(|\xi|), \overline{V(\xi)}=V(\xi), V(\xi) \in \mathcal{L}_{2, \text { lос }}\left(\mathbb{R}^{3}\right)$ и

$$
\lim _{|\xi| \rightarrow \infty} \int_{\left|\xi-\xi^{\prime}\right| \leqslant 1}\left|V\left(\xi^{\prime}\right)\right|^{2} d \xi^{\prime}=0 \text {. }
$$

Относительно магнитного вектор-потенциала предполагаем, что

$$
\begin{gathered}
\left|A_{\perp}\left(\rho_{1}\right)\right|^{2} \in \mathcal{L}_{2, \text { lос }}\left(\mathbb{R}^{2}\right), \\
\left|\frac{\partial A_{2}\left(\rho_{1}\right)}{\partial x_{1}}-\frac{\partial A_{1}\left(\rho_{1}\right)}{\partial y_{1}}\right| \rightarrow \infty \quad \text { при }\left|\rho_{1}\right| \rightarrow \infty .
\end{gathered}
$$

${ }^{1}$ Работа поддержана Международным научным фондом (грант № R94300) и Российским фондом фундаментальных исследований (грант № 94-01-01376).

${ }^{2}$ Которые выполняются, в частности, и тогда, когда частицы попарно не связываются. 
Если (2) не выполняется, то предполагаем (кроме (1)), что $A_{\perp}$ обладает $S O(2)$ симметрией, т. е. для некоторой функции $A\left(\left|\rho_{1}\right|\right)$

$$
A_{1}\left(\rho_{1}\right)=-y_{1} A\left(\left|\rho_{1}\right|\right), \quad A_{2}\left(\rho_{1}\right)=x_{1} A\left(\left|\rho_{1}\right|\right),
$$

и что

$$
\lim _{t \rightarrow \infty}|t A(t)|=\infty
$$

Условие (2) есть требование возрастания модуля вектора магнитного поля на бесконечности (в $(x, y)$-плоскости). Соотношение (4) выполняется как для возрастающего, так и для однородного или медленно убывающего поля.

Введем пространство $R_{z}$ относительного движения в направлении оси $z$ :

$$
R_{z}=\left\{\left(z_{1}, \ldots, z_{n}\right): \sum_{i=1}^{n} z_{i}=0\right\}
$$

и обозначим через $\Delta_{0 z}$ оператор $\frac{1}{m} \sum_{j=1}^{n} \frac{d^{2}}{d z_{j}^{2}}$ на $R_{z}$. Тогда, после отделения движения центра масс системы в направлении оси $z$, оператор энергии системы можно записать в виде ${ }^{3}$

$$
H_{N}=\sum_{t=1}^{N} \frac{1}{m}\left(i \nabla_{t \perp}+e A_{\perp}\left(\rho_{t}\right)\right)^{2}-\Delta_{0 z}+\frac{1}{2} \sum_{s, t, s \neq t}^{1, n} V\left(r_{s t}\right),
$$

где $\nabla_{t \perp}=\left(\partial / \partial x_{t}, \partial / \partial y_{t}\right)$.

Пусть $\mathbb{R}^{2 n}=\left\{\rho: \rho=\left(\rho_{1}, \ldots, \rho_{n}\right)\right\}, R_{0}=\mathbb{R}^{2 n} \oplus R_{z}$. Расширим оператор $H_{N}$ с области $C_{0}^{2}\left(R_{0}\right)$ до самосопряженного, сохраняя прежнее обозначение. Пусть $\alpha$ - допустимый (согласно принципу Паули) тип перестановочной симметрии системы, $P_{N}^{(\alpha)}$ - проектор в $\mathcal{L}_{2}\left(R_{0}\right)$ на подпространство функций симметрии $\alpha$ и $H_{N}^{(\alpha)}=P_{N}^{(\alpha)} H_{N}$. В ситуации (3) оператор $H_{N}$ инвариантен относительно вращений группы $S O(2)$ вокруг оси $z$. В этом случае обозначим через $H_{N}^{(m)}$, $H_{N}^{(\alpha, m)}$ сужения операторов $H_{N}, H_{N}^{(\alpha)}$ на подпространство функций, преобразующихся по представлению веса $m$ группы $S O(2)$.

Пусть $\sigma_{d}(H)$ - дискретный спектр произвольного оператора $H$. Будем говорить, что система $N$ частищ устойчива

в возрастающем магнитном поле, если $\sigma_{d}\left(H_{N}^{(\alpha)}\right) \neq \varnothing$ хотя бы для одного значения $\alpha$,

в невозрастающем магнитном поле, если $\sigma_{d}\left(H_{N}^{(\alpha, m)}\right) \neq \varnothing$ хотя бы для одной пары значений $\alpha, m$.

Разница в определении устойчивости для возрастающего и невозрастающего магнитных полей вызвана тем, что в невозрастающем магнитном поле для физически устойчивой системы возможно равенство $\sigma_{d}\left(H_{N}^{(\alpha)}\right)=\varnothing$ для любого $\alpha$ вследствие бесконечного вырождения по $m$ изолированных собственных значений в нижней

${ }^{3}$ Мы не пишем в (5) член, отвечающий взаимодействию спина с магнитным полем, ибо этот член не влияет на результаты (см. доказательство). 
части спектра. В то же время в этой ситуации $\sigma_{d}\left(H_{N}^{(\alpha, m)}\right) \neq \varnothing$ для какихлибо $\alpha, m$, т.е. физически устойчивая система устойчива и согласно нашему определению.

ТЕОРемА. Пусть потенциал $V(\xi)$ принадлежит $\mathcal{L}_{1}\left(\mathbb{R}^{3}\right)$ и

$$
\int V(\xi) d \xi<0
$$

Тогда существует такая бесконечная последовательность чисел $N_{p} \rightarrow \infty$, $p \rightarrow \infty$, что система $N_{p}$ частии устойчива.

СХемА дОКАЗАТЕЛЬСТВА. Для определенности будем предполагать, что рассматриваемые частицы имеют спин 1/2. Согласно [3], физически допустимые типы симметрии $\alpha=\alpha_{k}(N)$ для изучаемых систем отвечают разбиению числа $N$ на $k$ двоек и $N-2 k$ единиц, $k=0,1, \ldots,[N / 2]$ (т. е. двухстолбцовым схемам Юнга с первым столбщом из $N-k$ строк). Положим

$$
P_{N}(1 / 2)=\sum_{k=0}^{[N / 2]} P_{N}^{\left(\alpha_{k}\right)}, \quad \mathcal{H}_{N}=P_{N}(1 / 2) H_{N}, \quad \mathcal{H}_{N}^{(m)}=P_{N}(1 / 2) H_{N}^{(m)} .
$$

Таким образом, операторы $\mathcal{H}_{N}$ и $\mathcal{H}_{N}^{(m)}$ суть сужения операторов $H_{N}$ и $H_{N}^{(m)}$ на максимально широкие пространства функций, симметрия которых для рассматриваемых систем разрешена принципом Паули. Мы покажем, что дискретный спектр операторов $\mathcal{H}_{N}$ и $\mathcal{H}_{N}^{(m)}$ для всех $m$ не может быть пустым для всех $N \geqslant N_{0}$ ни при каком $N_{0}$. Доказательство основано на результатах работы авторов о локализации дискретного спектра многочастичных гамильтонианов в магнитных полях [4]. Оно опирается также на следующее свойство проектора $P_{N}(1 / 2)$ (вытекающее из леммы $\left.2.1[5]\right)$ : любая функция $\psi\left(r_{1}, \ldots, r_{N}\right)$ из пространства $P_{N}(1 / 2) C_{0}^{2}\left(\mathbb{R}^{3 N}\right)$, рассматриваемая как функция произвольных $3 n$ координат $r_{i_{1}}, \ldots, r_{i_{n}}, n<N$, при фиксированных значениях остальных переменных, принадлежит пространству $P_{n}(1 / 2) C_{0}^{2}\left(\mathbb{R}^{3 n}\right)$, где

$$
\mathbb{R}^{3 n}=\left\{\left(r_{i_{1}}, \ldots, r_{i_{n}}\right)\right\} .
$$

Отметим, что, в отличие от оператора $P_{N}(1 / 2)$, ни один из составляющих его операторов $P_{N}^{\left(\alpha_{k}\right)}$ (кроме оператора, отвечающего антисимметричному представлению) не обладает указанным свойством и именно в этом причина наличия в теореме 2.2 из [2] неравенств (3.1), отсутствующих здесь.

Предположение о том, что $\sigma_{d}\left(\mathcal{H}_{N}\right)=\varnothing$ и что в ситуации $(3) \sigma_{d}\left(\mathcal{H}_{N}^{(m)}\right)=\varnothing$ для любого $m$ при $N \geqslant N_{0}$ для некоторого $N_{0}$, приводит в силу $[4,5,2]$ к неравенствам

$$
\inf \mathcal{H}_{N} \geqslant-d_{0} N, \quad \inf \mathcal{H}_{N}^{(m)} \geqslant-d_{0} N \quad \text { для любого } m,
$$

где $d_{0}$ - некоторая константа, не зависящая от $N$. В то же время рассмотрение предложенных в [2, с. 135] семейств пробных функций показывает, что

$$
\inf \mathcal{H}_{N} \leqslant-d_{1} N^{2}
$$

где $d_{1}$ - константа, не зависящая от $N$. (Здесь учтено, что для этих пробных функций энергия системы, отвечающая магнитному полю и взаимодействию магнитного поля со спином, не может расти быстрее чем $d_{3} N$ с константой $d_{3}$, не 
зависящей от $N$.) Так как при условии (3) $\mathcal{H}_{N}=\bigoplus_{m=-\infty}^{+\infty} \mathcal{H}_{N}^{(m)}$, то для каждого $N$ можно найти такое число $m=m_{N}$, что

$$
\inf \mathcal{H}_{N}^{\left(m_{N}\right)} \leqslant-d_{1} N^{2} .
$$

Неравенства (9), (10) противоречат неравенствам (8), и, следовательно, предположения о том, что $\sigma_{d}\left(\mathcal{H}_{N}\right)=\varnothing$ или, в ситуации $(3), \sigma_{d}\left(\mathcal{H}_{N}^{(m)}\right)=\varnothing$ для любого $m$ при любых больших $N$, не верны, т. е. теорема доказана.

\section{ЛИтЕРАТУРА}

1. Avron J., Herbst I., Simon B. Ann. Phys., 114, No. 4, 431 (1978). 2. Вугальтер C. А., Жислин Г. М. Теор. матем. физ., 76, №1, 132-142 (1988). 3. Вигнер $E$. Теория групп и ее приложения к квантово-механической теории атомных спектров. ИЛ, М., 1961. 4. Вугальтер С. А., Жислин Г. М. Теор. матем. физ., 97, №1, 94-112 (1993). 5. Жислин Г. М., Сигалов А. Г. Изв. АН СССР, сер. матем., 29, №4, 835-860 (1965).

Научно-исследовательский радиофизический институт, Н. Новгород

Поступило в редакцию 25 апреля 1994 г.

УдК $517.986 .9+515.165 .4$

\section{Квадратичные соотношения в когомологиях обобщенных крашеных кос и тождества Данкла}

(c) 1996. В. А. ГолУБевА, В. П. ЛЕксин ${ }^{1}$

Пусть $A=\bigcup_{1 \leqslant i<j \leqslant n}\left\{z_{i}-z_{j}=0\right\}$ - связка гиперплоскостей, ассоциированная с системой корней типа $A_{n-1}$, в комплексном аффинном пространстве $\mathbb{C}^{n}$ и $Y_{n}=\mathbb{C}^{n} \backslash A$ - дополнение к ней типа $K(\pi, 1)$. Группа крашеных кос $P_{n}$ - это фундаментальная группа пространства $Y_{n}$, и для ее когомологий имеет место изоморфизм

$$
H^{*}\left(P_{n}, \mathbb{Z}\right)=H^{*}\left(Y_{n}, \mathbb{Z}\right) .
$$

В работе [1] В. И. Арнольд показал, что когомологии $H^{*}\left(Y_{n}, \mathbb{Z}\right)$ изоморфны подкольцу $\Omega(n)$ внешних форм на $Y_{n}$, порожденному над $\mathbb{Z}$ единицей и формами $\omega_{i j}=d\left(z_{i}-z_{j}\right) /\left(z_{i}-z_{j}\right), i<j$. Там же дано комбинаторное описание этого кольца как внешней алгебры $\Lambda(n)$ с образующими $\omega_{i j}=\omega_{j i}, 1 \leqslant i<j \leqslant n$, и квадратичными соотношениями

$$
\omega_{i j} \wedge \omega_{j k}+\omega_{j k} \wedge \omega_{i k}+\omega_{i k} \wedge \omega_{i j}=0 .
$$

Э. Брискорн в работе [2] доказал, что для любых конечных аффинных связок гиперплоскостей $S=\bigcup_{i \in I} H_{i}$ в $\mathbb{C}^{n}$ когомологии пространства $Y_{n}(S)=\mathbb{C}^{n} \backslash S$ изоморфны подкольцу $\Omega_{n}(S)$ в алгебре внешних форм на $Y_{n}(S)$, порожденному

${ }^{1}$ Работа выполнена при частичной под держке ISF, грант М3С300, РФФИ и Российского правительства. 